\title{
Fundamenta Informaticae (2009) Petri Nets 2008
}

\section{Preface}

It is a pleasure to present a selection from the best papers of the 29th International Conference on Application and Theory of Petri Nets and Other Models of Concurrency (PETRI NETS 2008), held in Xi' an, China, June 23-27, 2008. The Petri Net conference has already a long tradition and is well-known among the best theoreticians in computer science and also by people involved in practical applications. For more information about the Petri net conferences and related activities see: http://www.informatik.unihamburg.de/TGI/PetriNets/ The Petri Net conference serves as the annual meeting place for researchers to exchange and to discuss the new results in the field of Petri Nets and other models of concurrency. Not only theoretical results are presented but also innovative applications. Novel tools, or substantial enhancements of existing tools for modeling, analysis or enactment of Petri Nets are presented as well. The conference always has a number of invited talks in which surveys on related fields are presented. The conference is always accompanied by workshops and tutorials. The 2008 conference had five invited speakers, two tutorials, four workshops and there was a tool exhibition. The conference received 75 submissions by authors from 21 different countries. The Program Committee accepted 23 papers, classified as: 14 theory papers, 5 application papers and 4 tool papers. The Petri Nets 2008 conference was organized by the Institute of Computing Theory and Technology at Xidian University, Xi' an, China. The Organizing Committee, chaired by Zhenhua Duan, did a very good job. This is the second time that the best papers presented at the conference are selected for a special issue of Fundamenta Informaticae. The authors of the selected papers were invited to submit substantially enhanced versions of their papers, which means that these papers differ significantly from the original conference papers. Each paper was reviewed according to the standards of the journal and if necessary revised by the authors.

The seven selected papers in this special issue cover a variety of new results in theory as well as in applications. The paper "Synthesis of nets with step firing policies", introduces a way to control step semantics in order to restrict the concurrent behavior to improve modeling features. They also provide algorithms to synthesize a Petri net out of transition systems controlled by such a step firing policy. The paper "A net-based approach to Web services publication and replaceability" addresses the problem of interchangeability of services in arbitrary environments, based on an appropriate notion of behavioral equivalence. Further the paper introduces an ontology-based way of specifying services. In paper "Unfolding semantics of Petri nets based on token flows" two new unfolding semantics are presented that still 
represent the complete partial order behavior. Also algorithms are developed for complete finite prefixes of both unfolding models. These turn out to be much smaller and and are faster to compute. In paper "Modelling and validation of secure connection establishment in a generic access network scenario" a generic CPN model for telephone services via a generic IP network is described, in particular the Internet protocols IPSec and IKEv2 are modeled. The models are used as formal specification and for validation of it. The paper "Process discovery using integer linear programming" gives a method to construct a Petri net out of a finite set of traces, assumed to be coming from an execution log. The constructed Petri net is able to reproduce the trace set and is independent of the number of process instances in the log. The approach is based on the language-based theory of regions and applies standard integer linear programming techniques. In paper "Building efficient model checkers using hierarchical set decision diagrams and automatic saturation" a new approach is presented to deal with state-space explosion for model checking, based on set-labeled decision diagrams in which the arcs are labeled with sets, which are themselves stored as set-labeled decision diagrams. The method is very efficient which is illustrated by convincing examples. In the paper "A practical approach to verification of mobile systems using net unfoldings" finite control processes, well-known from $\pi$-Calculus are translated into safe Petri nets, for which efficient unfolding-based verification is possible. Experiments show that this approach is much more efficient than existing methods for verification of these systems.

We like to express our gratitude to all authors for the excellent quality of the papers in this special issue. We also like to thank the reviewers of these papers for their hard work and constructive comments.

\section{Special issue editors}

Kees van Hee and Rüdiger Valk

Petri Nets 2008 Program Co-Chairs

Eindhoven, Hamburg August 2009 